\title{
Does amantadine induce acute psychosis? A case report and literature review
}

This article was published in the following Dove Press journal:

Neuropsychiatric Disease and Treatment

6 April 2016

Number of times this article has been viewed

\author{
Wei-juan $X u^{1,2}$ \\ Ning Wei ${ }^{1,2}$ \\ Yi Xu ${ }^{1,2}$ \\ Shao-hua $\mathrm{Hu}^{1,2}$ \\ 'Department of Psychiatry, The \\ First Affiliated Hospital of Zhejiang \\ University School of Medicine, ${ }^{2}$ The \\ Key Laboratory of Mental Disorder's \\ Management of Zhejiang Province, \\ Hangzhou, People's Republic of China
}

Correspondence: Shao-hua Hu Department of Psychiatry, The First Affiliated Hospital of Zhejiang University School of Medicine, No 79, Qingchun Road, Hangzhou 310003, Zhejiang, People's Republic of China

Tel +86 57I 56723001

Email dorhushaohua@zju.edu.cn
Background: Over-the-counter cold medicines, which contain amantadine, are widely used in the People's Republic of China. Clinicians are familiar with the psychosis caused by longterm treatment with amantadine, especially in elderly patients; however, early-onset psychotic complications among healthy young individuals have rarely been reported.

Case presentation: This article reports the case of a 28 -year-old patient who presented with hallucination-delusion syndrome soon after treatment with cold medicine containing amantadine hydrochloride and acetaminophen. The symptoms resolved completely after a 2 -week course of paliperidone treatment.

Conclusion: Clinicians should be sensitive to the acute psychotic complications induced by an interaction between amantadine and acetaminophen.

Keywords: amantadine, acetaminophen, side effects

\section{Background}

Over-the-counter cold medicine, which contains amantadine, is widely used in the People's Republic of China. Psychosis and delirium induced by amantadine have been previously reported among elderly patients. Typically, it takes several months of amantadine administration to bring about these side effects. ${ }^{1}$ In this article, we report on the case of a 28-year-old patient suffering from a cold who presented with hallucination-delusion syndrome immediately after the use of a cold medicine containing amantadine hydrochloride and acetaminophen.

\section{Case presentation}

A 28-year-old man was admitted to The First Affiliated Hospital of Zhejiang University School of Medicine because of hallucinations and persecutory delusions, which lasted for $\sim 48$ hours. When he was alone at home, he had an auditory hallucination that his parents described of inviting a Taoist, and he "heard" that his birthday horoscope did not match with that prepared by his parents. The patient reported that he could sometimes "see" the Taoist. The auditory hallucinations lasted for almost an entire day and severely affected the patient's sleep. He simultaneously expressed fear that someone wanted to control and harm him. He did not report experiencing headache, fever, or convulsions. Prior to admission, he had been in excellent health and did not have a history of medical problems, psychiatric disorders, or substance abuse. He was born in Zhejiang province of the People's Republic of China and had not traveled elsewhere. He was single and did not report recent sexual contact. He was a nonsmoker and did not drink alcohol habitually. His family history was negative for psychotic disorders. 
Five days prior to admission, he reported symptoms of a cold, including a runny nose, and had therefore started taking an over-the-counter cold medicine (brand name: Kuaike), which contained $250 \mathrm{mg}$ of acetaminophen and $100 \mathrm{mg}$ of amantadine hydrochloride. The patient took one capsule twice daily. Three days later, he began to experience hallucinations and delusions.

During the mental status examination administered upon admission, the patient was described as a slightly thin and cooperative man. The patient was fully oriented to person, place, and time. However, he frequently looked down and did not maintain eye contact with the examiner. His speech was a bit slow, but with normal tone, rhythm, and prosody. He had obvious auditory and visual hallucinations. His thought process was linear; however, he expressed persecutory delusions. His mood was anxious and congruent. No suicidal or homicidal ideation was reported or exhibited. Although the patient acknowledged his own abnormal mental state, his insight was still partially impaired.

Physical and neurologic examinations did not identify any abnormalities. Laboratory studies revealed complete blood count, electrolytes, glucose, urea, creatinine, hepatic function, and thyroid hormone levels within normal limits, and the rapid plasma reagin test, serum human immunodeficiency virus (HIV) antibody test, and urinalysis for narcotic drugs were negative. Electroencephalogram (EEG) and cerebral magnetic resonance imaging (MRI) appeared normal.

The patient was immediately treated with haloperidol (5 mg daily) and paliperidone extended release (3 mg daily), while Kuaike administration was ceased. After 48 hours of treatment, his symptoms were markedly improved. Auditory and visual hallucinations, as well as paranoid ideation, resolved completely. Haloperidol was used for 3 days, and the use of paliperidone extended release was maintained for 2 weeks to prevent relapse. After the 2 -week treatment course, he had fully recovered and was discharged. Six months later, we conducted a telephonic follow-up, wherein the patient reported of no subsequent psychotic experiences.

\section{Ethics approval and consent}

Written informed consent was obtained from the patient for the publication of this case report. A copy of the written consent is available for review. The report was approved by The First Affiliated Hospital of Zhejiang University School of Medicine ethics committee.

\section{Discussion}

Existing literature reports about several drugs that could induce acute psychosis, such as psychostimulants, ${ }^{2,3}$ antibiotics, ${ }^{4}$ antivirals, ${ }^{5}$ and antiparkinsonism. ${ }^{6}$ Amantadine is indicated for the treatment of influenza A, parkinsonism, and drug-induced extrapyramidal reactions. The mechanism of amantadine action in the central nervous system is not well understood. Increasing evidence shows that amantadine enhances dopamine release indirectly via antagonism of the N-methyl-D-aspartate receptor, and this mechanism may be responsible for this rarely exhibited psychotic side effect. ${ }^{7}$ N-methyl-D-aspartate antagonists, such as ketamine, can induce the positive, negative, and cognitive symptoms of schizophrenia. ${ }^{8}$

There are numerous reports of amantadine being prescribed for long-term antiparkinsonian effect., ${ }^{9,10}$ However, to our knowledge, there were only three previous reports of rapid psychiatric complications among otherwise healthy and young individuals. The first report described an acute psychosis secondary to an amantadine overdose, ${ }^{11}$ the second noted the emergence of psychosis following $100 \mathrm{mg}$ of amantadine twice daily in combination with venlafaxine and quetiapine, ${ }^{12}$ and the third study presented two cases of psychosis among 295 subjects in an antiviral trial of amantadine. ${ }^{13}$

The current case demonstrates the potential adverse effects of amantadine on the central nervous system among young healthy adults at the standard dose. Although the incidence of this induced psychosis is low, it may increase with the daily administration of amantadine, and clinicians should be aware of the rapid onset of these psychotic complications. Recently, the use of amantadine in the treatment of influenza A has been discouraged. ${ }^{14}$ Because of its limited effectiveness, amantadine is only recommended for use during a serious epidemic or pandemic alongside other public health measures. ${ }^{15}$

Although we believe that amantadine was the primary cause of this patient's psychosis, we cannot exclude the effects acetaminophen might have had in this adverse reaction. There have been only two reported cases of acetaminophen use associated with psychosis: acetaminophen in combination with codeine and acetaminophen overdose. ${ }^{16,17}$ Acetaminophen has been reported to have an inhibitory effect on prostaglandin synthase in the brain, ${ }^{18}$ and prostaglandin itself has been linked to the etiology of schizophrenia. ${ }^{19}$

\section{Conclusion}

Over-the-counter cold medicines containing amantadine are widely used in the People's Republic of China. Clinicians should be aware of the severe side effects of these medicines on the central nervous system. 


\section{Acknowledgment}

This work was supported by a grant from the National Science and Technology Program (2015BAI13B02).

\section{Author contributions}

All authors contributed toward data analysis, drafting and critically revising the paper and agree to be accountable for all aspects of the work.

\section{Disclosure}

The authors report no conflicts of interest in this work.

\section{References}

1. Harper RW, Knothe BU. Coloured Lilliputian hallucinations with amantadine. Med J Aust. 1973;1(9):444-445.

2. Kraemer M, Uekermann J, Wiltfang J, Kis B. Methylphenidate-induced psychosis in adult attention-deficit/hyperactivity disorder: report of 3 new cases and review of the literature. Clin Neuropharmacol. 2010; 33(4):204-206.

3. Grau-Lopez L, Roncero C, Navarro MC, Casas M. Psychosis induced by the interaction between disulfiram and methylphenidate may be dose dependent. Subst Abus. 2012;33(2):186-188

4. Kouvelou E, Pourzitaki C, Aroni F, Papazisis G, Kouvelas D. Acute psychosis induced by clarithromycin in a healthy adult? J Clin Psychopharmacol. 2008;28(5):579-580.

5. Cheng YC, Chen CC, Ho AS, Chiu NY. Prolonged psychosis associated with interferon therapy in a patient with hepatitis $\mathrm{C}$ : case study and literature review. Psychosomatics. 2009;50(5):538-542.

6. Signorelli MS, Battaglia E, Costanzo MC, Cannavo D. Pramipexole induced psychosis in a patient with restless legs syndrome. BMJ Case Rep. 2013;2013:bcr2013009716.

7. Blanpied TA, Clarke RJ, Johnson JW. Amantadine inhibits NMDA receptors by accelerating channel closure during channel block. J Neurosci. 2005;25(13):3312-3322.
8. Krystal JH, Perry EB Jr, Gueorguieva R, et al. Comparative and interactive human psychopharmacologic effects of ketamine and amphetamine: implications for glutamatergic and dopaminergic model psychoses and cognitive function. Arch Gen Psychiatry. 2005;62(9):985-994.

9. Stefanis N, Bozi M, Christodoulou C, et al. Isolated delusional syndrome in Parkinson's disease. Parkinsonism Relat Disord. 2010;16(8): $550-552$

10. Poewe W. When a Parkinson's disease patient starts to hallucinate. Pract Neurol. 2008;8(4):238-241.

11. Snoey ER, Bessen HA. Acute psychosis after amantadine overdose. Ann Emerg Med. 1990;19(6):668-670.

12. Smith EJ. Amantadine-induced psychosis in a young healthy patient. Am J Psychiatry. 2008;165(12):1613.

13. Flaherty JA, Bellur SN. Mental side effects of amantadine therapy: its spectrum and characteristics in a normal population. J Clin Psychiatry. 1981;42(9):344-345.

14. Centers for Disease Control and Prevention [webpage on the Internet] National Center for Immunization and Respiratory Diseases: Seasonal Influenza Antiviral Dosage. Centers for Disease Control and Prevention. Available from: http://www.cdc.gov/flu/professionals/antivirals/ antiviral-dosage.htm. Accessed November 30, 2015.

15. Jefferson T, Demicheli V, Rivetti D, Jones M, Di Pietrantonj C, Rivetti A. Antivirals for influenza in healthy adults: systematic review. Lancet. 2006;367(9507):303-313.

16. Malek-Ahmadi P, Ramsey ML. Acute psychosis associated with codeine and acetaminophen: a case report. Neurobehav Toxicol Teratol. 1985;7(2):193-194.

17. Servis M, Connolly B. Acute psychosis associated with acetaminophen overdose. Gen Hosp Psychiatry. 1997;19(2):149-150.

18. Flower RJ, Vane JR. Inhibition of prostaglandin synthetase in brain explains the anti-pyretic activity of paracetamol (4-acetamidophenol) Nature. 1972;240(5381):410-411.

19. Heleniak E, O’Desky I. Histamine and prostaglandins in schizophrenia: revisited. Med Hypotheses. 1999;52(1):37-42.
Neuropsychiatric Disease and Treatment

\section{Publish your work in this journal}

Neuropsychiatric Disease and Treatment is an international, peerreviewed journal of clinical therapeutics and pharmacology focusing on concise rapid reporting of clinical or pre-clinical studies on a range of neuropsychiatric and neurological disorders. This journal is indexed on PubMed Central, the 'PsycINFO' database and CAS,

\section{Dovepress}

and is the official journal of The International Neuropsychiatric Association (INA). The manuscript management system is completely online and includes a very quick and fair peer-review system, which is all easy to use. Visit http://www.dovepress.com/testimonials.php to read real quotes from published authors. 